Communications in Physics, Vol. 20, No. 3 (2010), pp. 193-200

\title{
MOBILITY ENHANCEMENT IN SQUARE QUANTUM WELLS: SYMMETRIC MODULATION OF THE ENVELOP WAVE FUNCTION
}

\author{
DOAN NHAT QUANG \\ Center for Theoretical Physics, Institute of Physics, VAST \\ NGUYEN HUYEN TUNG AND NGUYEN TRUNG HONG \\ Institute of Engineering Physics, Hanoi University of Technology \\ TRAN THI HAI \\ Department of Engineering and Technology, Hong Duc University
}

\begin{abstract}
We present a theoretical study of the effects from symmetric modulation of the envelop wave function on quantum transport in square quantum wells ( $Q W s$ ). Within the variational approach we obtain analytic expressions for the carrier distribution and their scattering in symmetric two-side doped square QWs. Roughness-induced scattering are found significantly weaker than those in the asymmetric one-side doped counterpart. Thus, we propose symmetric modulation of the wave function as an efficient method for enhancement of the roughness-limited $Q W$ mobility. Our theory is able to well reproduce the recent experimental data about low-temperature transport of electrons and holes in two-side doped square QWs, e.g., the mobility dependence on the channel width, which have not been explained so far.
\end{abstract}

\section{INTRODUCTION}

Realization of conducting channels with high mobility is one of the most challenging subjects in semiconductor physics and is important for device applications. For improving device's performance, it is required not only to increase carrier density but also to increase carrier mobility. Large mobility enables fabrication of devices of high current drive and high switching speed. As known, [1] enhanced mobility of two-dimensional (2D) carriers in quantum wells (QWs) is achieved by means of modulation of the decisive factors such as electronic structure, scattering mechanisms and confining sources.

So far, various methods for modulation have been proposed. The strain present in lattice-mismatched QWs is shown [2] to result in a modulation of their electronic structures that the in-plane effective mass is reduced. Accordingly, there have been several experimental attempts to grow higher mobility in 2D strained channels for electrons in Si, [4] holes in GaAs, [5] Ge, [6,7] and Si. [8] Another method combines the effects on Ge from strain and alloying with Sn to reduce the in-plane and density-of-state masses, [9] so increasing phonon-limited mobilities of both electrons and holes in Ge. As to scattering mechanisms, the modulation doping is regarded as a successful technique for reducing the impurity scattering. [10] As known, [1] the impact of a scattering event in the in-plane depends strongly on the envelop wave function along the quantization direction, which is 
shaped by confining sources. So, it was suggested [11] that this function is modulated by insertion of thin barrier layers into the channel for reducing the phonon impact. However, it should be mentioned that with the quoted methods, additional scatterings are implied, e.g., alloy disorder [12] and misfit deformation potential, [13,14] which may cancel their merits.

Recently, we have shown $[15,16]$ that asymmetric modulation of the envelop wave function, e.g., by a single-side doping of QWs, may increase remarkably the impact of roughness-related scatterings, viz., surface roughness and misfit deformation potential, so the mobility degraded drastically. Therefore, we suggest a possibility for enhanced mobility via symmetric modulation of the wave function, e.g., by double-side doping. So far, there have been a number of experimental reports $[17,19,21-26]$ on the transport properties of double-side doped square QWs based, e.g., on relaxed-GaAs and strained-Ge channels, however, no theoretical analysis available.

Thus, the aim of this paper is to present a theoretical study of low-temperature transport in the strained channel of symmetrically-modulated square QWs. The theory is developed within the variational approach to the band-bending effects from doubleside modulation doping. Further, the theory includes all possible scattering mechanisms, especially misfit deformation potential.

In Sec. II, we supply the basic equations for calculating the distribution of carriers along the growth direction. In Sec. III, their mobility in the in-plane is calculated. Numerical results and comparison with experimental data are presented in Sec. IV.

\section{SYMMETRIC TWO-SIDE DOPED SQUARE QW}

\section{II.1. Variational Wave Function}

To start with, we examine the effect from doping-induced band bending on the carrier distribution along the growth direction. The doping profile is regarded as symmetric if there are two doped layers symmetric with respect to the channel center $(z=0)$, i.e., with an equal doping density and an equal doping geometry (doping and spacer layers). With equal potential barriers the $2 \mathrm{~S}$ doped square QW is a completely symmetric system. Therefore, for high enough barriers, we may take a symmetric envelop wave function for carriers (electrons or heavy holes) in the lowest subband of the QW as follows:

$$
\zeta(z)= \begin{cases}2 B \sqrt{\pi / L} \cos (\pi z / L) \cosh (c z / L), & \text { for }|z| \leq L / 2 \\ 0, & \text { for }|z|>L / 2\end{cases}
$$

with $L$ as the well width. The normalization requires that $\pi B^{2}\left[\gamma_{1}(c)+1\right]=1$ with $n=0,1,2, \ldots$ as an integer. Where $\gamma_{n}(x)$ with $n$ as an integer is a simple function defined by Eq. (2) below.

$$
\gamma_{n}(x)=\left[\frac{1}{x}+\frac{(-1)^{n} x}{x^{2}+n^{2} \pi^{2}}\right] \sinh x
$$

Thus, there is a single independent parameter, say $c$, which is a measure of the bandbending effect from $2 \mathrm{~S}$ doping on the carrier distribution. 


\section{LOW-TEMPERATURE MOBILITY}

Within the linear transport theory, the mobility at very low temperatures are determined by the transport lifetime: $\mu=e \tau / m^{*}$, with $m^{*}$ as the in-plane effective mass of the carrier. The transport lifetime is represented in terms of the autocorrelation function $(\mathrm{ACF})$ for each disorder by [29]

$$
\frac{1}{\tau}=\frac{1}{(2 \pi)^{2} \hbar E_{F}} \int_{0}^{2 k_{F}} d q \int_{0}^{2 \pi} d \varphi \frac{q^{2}}{\left(4 k_{F}^{2}-q^{2}\right)^{1 / 2}} \frac{\left\langle|U(\mathbf{q})|^{2}\right\rangle}{\varepsilon^{2}(q)} .
$$

Here $\mathbf{q}=(q, \varphi)$ is the $2 \mathrm{D}$ momentum transfer due to a scattering event in the $x$-y plane (in polar coordinates): $q=|\mathbf{q}|=2 k_{F} \sin (\vartheta / 2)$ with $\vartheta$ as a scattering angle. The Fermi energy is given by $E_{F}=\hbar^{2} k_{F}^{2} / 2 m^{*}$, with $k_{F}=\sqrt{2 \pi p_{s}}$ as the Fermi wave number and $p_{s}$ is the sheet density. The dielectric function $\varepsilon(q)$ in Eq. (3) takes account of the screening of a scattering potential by the carriers. The ACF in Eq. (3), $\left\langle|U(\mathbf{q})|^{2}\right\rangle$, is defined by an ensemble average of the 2D Fourier transform of the (unscreened) scattering potential weighted with an envelop wave function. The carriers are expected to be subject to the following scattering mechanisms: (i) remote impurity (RI), (ii) surface roughness (SR), and (iii) misfit deformation potential (DP). The overall lifetime $\tau_{\text {tot }}$ is then determined by the ones for individual disorders according to the Matthiessen rule,

$$
\frac{1}{\tau_{\text {tot }}}=\frac{2}{\tau_{\mathrm{RI}}}+\frac{2}{\tau_{\mathrm{SR}}}+\frac{2}{\tau_{\mathrm{DP}}}
$$

where a factor of 2 is inserted to allow for equal scatterings from two doping layers and two rough interfaces.

\section{AUTOCORRELATION FUNCTIONS FOR SCATTERING MECHANISMS}

\section{IV.1. Remote Impurity}

According to Eq. (3), to evaluate the transport lifetime we ought to derive the ACFs for the above-quoted scattering sources. First, the ACF for scattering from a random distribution of remote impurities is supplied in terms of an integral over the doping layer, $[29]$

$$
\left\langle\left|U_{\mathrm{RI}}(\mathbf{q})\right|^{2}\right\rangle=\left(\frac{2 \pi e^{2}}{\varepsilon_{L} q}\right)^{2} \int_{-\infty}^{+\infty} d z_{i} N_{I}\left(z_{i}\right) F_{R}^{2}\left(q, z_{i}\right) .
$$

Here, $N_{I}\left(z_{i}\right)$ is the impurity distribution and $F_{R}\left(q, z_{i}\right)$ is the form factor for an impurity sheet located at the plane $z=z_{i}$, given by

$$
F_{R}\left(q, z_{i}\right)=\int_{-\infty}^{+\infty} d z|\zeta(z)|^{2} e^{-q\left|z-z_{i}\right|}
$$

We may arrive at the ACF for correlated remote impurities in the form:

$$
\left\langle\left|U_{\mathrm{RI}}(\mathbf{q})\right|^{2}\right\rangle_{c}=\left(\frac{2 \pi e^{2}}{\varepsilon_{L}}\right)^{2} \frac{N_{I} L^{3}}{4} F_{\mathrm{RI}}(t),
$$


where the scattering form factor is

$$
F_{\mathrm{RI}}(t)=\frac{R^{2}(t)}{2} \frac{e^{-2 s t}-e^{-2 d t}}{t^{2}\left(t+t_{c}\right)}
$$

Here, we introduce a function of the dimensionless momentum transfer $t=q L$ by

$$
R(t)=\pi B^{2}\left[\gamma_{1}(c+t / 2)+\gamma_{1}(c-t / 2)+2 \gamma_{1}(t / 2)\right]
$$

with $\gamma_{1}(x)$ given by Eq. (2), the correlation parameter is given by

$$
q_{c}=\frac{2 \pi e^{2} N_{I}^{2 D}}{\varepsilon_{L} k_{B} T_{0}}
$$

\section{IV.2. Surface Roughness}

Next, we derive the ACF for surface roughness scattering. The weighted potential in wave vector space for SR scattering from the top interface is given in terms of the local value of the wave function $\zeta_{-}=\zeta(z=-L / 2)$ by [1]

$$
U_{\mathrm{SR}}(\mathbf{q})=V_{0}\left|\zeta_{-}\right|^{2} \Delta_{\mathbf{q}}
$$

where $\Delta_{\mathbf{q}}$ is a Fourier transform of the roughness profile, $E(c)$ is the total energy per particle in the lowest subband, $V_{H}(0)$ is the Hartree potential at $z=0, \varepsilon_{L}$ is the dielectric constant.

$$
\begin{aligned}
V_{0}\left|\zeta_{-}\right|^{2}=\left[E(c)-V_{H}(0)\right] \zeta^{2}(0)+\frac{\pi^{3} e^{2} B^{4} p_{s}}{2 \varepsilon_{L}}\left\{\frac { 1 } { c ^ { 2 } + \pi ^ { 2 } } \left[\frac{2 c^{2}+\pi^{2}}{c}\left[\theta_{1}(2 c)+2 \theta_{1}(c)\right]\right.\right. \\
+\frac{c}{2}\left[\theta_{2}(2 c)+2 \theta_{2}(c)-\theta_{0}(2 c)-2 \theta_{0}(c)\right]-\frac{\pi}{2}\left[\sigma_{2}(2 c)+2 \sigma_{1}(2 c)\right]-\frac{c^{2}+2 \pi^{2}}{\pi} \\
\left.\left.\times\left[\sigma_{2}(c)+2 \sigma_{1}(c)\right]-\frac{2 c^{2}+3 \pi^{2}}{2 \pi}\left[\sigma_{2}(0)+2 \sigma_{1}(0)\right]\right]+2\left[\frac{\partial \theta_{1}(c)}{\partial c}+\frac{\partial \theta_{1}(0)}{\partial c}\right]\right\}
\end{aligned}
$$

where we introduce the following auxiliary functions:

$$
\begin{array}{r}
\theta_{n}(x)=\frac{\cosh x-1}{x}+\frac{x}{x^{2}+n^{2} \pi^{2}}\left[(-1)^{n} \cosh x-1\right] \\
\sigma_{n}(x)=\frac{n \pi}{x^{2}+n^{2} \pi^{2}}\left[(-1)^{n} \cosh x-1\right]
\end{array}
$$

\section{IV.3. Misfit Deformation Potential}

Lastly, it is well known $[28,30]$ that in lattice-mismatched QWs, e.g., of SiGe/Ge/SiGe, the well layer is subject to some strain. For pseudomorphic QWs, the in-plane component of the strain field in the well is specified by the misfit defined as

$$
\epsilon_{\|}=\frac{a_{\|}-a_{0}}{a_{0}}
$$

where $a_{\|}$and $a_{0}$ are the lattice constants of this layer in the presence and in the absence of strain, respectively. We supply the $2 \mathrm{D}$ Fourier transform of the misfit DP for cubic 
crystals. The scattering potential associated with the top interface $(z=-L / 2)$ is given as follows for electrons: $[13,14]$

$$
\left\langle\left|U_{\mathrm{DP}}^{(e)}(\mathbf{q})\right|^{2}\right\rangle=\left(\frac{\pi^{3 / 2} \alpha \epsilon_{\|} \Xi_{u}(K+1) \Delta \Lambda B^{2}}{4 L}\right)^{2} F_{D P}(t)
$$

and for holes:

$$
\begin{array}{r}
\left\langle\left|U_{\mathrm{DP}}^{(h)}(\mathbf{q})\right|^{2}\right\rangle=\left(\frac{\pi^{3 / 2} \alpha \epsilon_{\|} \Delta \Lambda B^{2}}{4 L}\right)^{2} F_{D P}(t) \times\left\{\frac{3}{2}\left[b_{s}(K+1)\right]^{2}\right. \\
\left.\left(1+\sin ^{4} \varphi+\cos ^{4} \varphi\right)+\left(\frac{d_{s} G}{4 c_{44}}\right)^{2}\left(1+\sin ^{2} \varphi \cos ^{2} \varphi\right)\right\} .
\end{array}
$$

Here, the relevant scattering form factor is given by

$$
F_{\mathrm{DP}}(t)=t^{2} e^{-t}\left[\gamma_{1}(c+t / 2)+\gamma_{1}(c-t / 2)+2 \gamma_{1}(t / 2)\right]^{2} \frac{1}{\left(1+\lambda^{2} t^{2} / 4 n\right)^{n+1}} .
$$

Here, $\Xi_{u}$ is the DP constant for the conduction band of the well, $b_{s}$ and $d_{s}$ are those for its valence band. The anisotropy ratio is yielded by

$$
\alpha=2 \frac{c_{44}}{c_{11}-c_{12}},
$$

the elastic constants by

$$
K=2 \frac{c_{12}}{c_{11}}, \quad G=2\left(c_{11}+2 c_{12}\right)\left(1-\frac{c_{12}}{c_{11}}\right),
$$

with $c_{i j}$ as elastic stiffness constants of the well.

\section{RESULTS AND CONCLUSIONS}

In this section, we apply the above-developed theory in order to understand the properties of low-temperature transport in remote doped square QWs. In particular, we clarify the advantage of symmetric $2 \mathrm{~S}$ doping over asymmetric $1 \mathrm{~S}$ doping. To illustrate, we introduce the enhancement factor defined by the ratio of the overall mobility in the $2 \mathrm{~S}$ doped QW $\left(\mu_{\mathrm{tot}}^{2 \mathrm{~S}}\right)$ to that in the $1 \mathrm{~S}$ doped counterpart $\left(\mu_{\mathrm{tot}}^{1 \mathrm{~S}}\right)$ under the same doping and interface profiles,

$$
Q\left(L, p_{s} ; \Lambda\right)=\frac{\mu_{\mathrm{tot}}^{2 \mathrm{~S}}\left(L, p_{s} ; \Delta, \Lambda\right)}{\mu_{\mathrm{tot}}^{1 \mathrm{~S}}\left(L, p_{s} ; \Delta, \Lambda\right)} .
$$

We now explore the above functional dependence. As an example, the numerical calculations are performed for the $p$-type $\mathrm{Si}_{0.3} \mathrm{Ge}_{0.7} / \mathrm{Ge} / \mathrm{Si}_{0.3} \mathrm{Ge}_{0.7}$ square QW. The overall mobilities entering in Eq. (21) are estimated in accordance with the Matthiessen rule. The SR and misfit DP partial mobilities in the symmetric 2S doped QW are calculated by employing Eqs. (12) and (17), while the relevant mobilities in the asymmetric 1S doped QW were calculated in Ref. [16]. Where the correlation length is given with $\Lambda=75 \AA$, the enhancement factor $Q$ is plotted versus (a) the well width $L$ for various hole densities $p_{s}=10^{11}, 10^{12}, 10^{13} \mathrm{~cm}^{-2}$, and (b) the sheet hole density $p_{s}$ for various well widths $L=75,150,300 \AA$. 

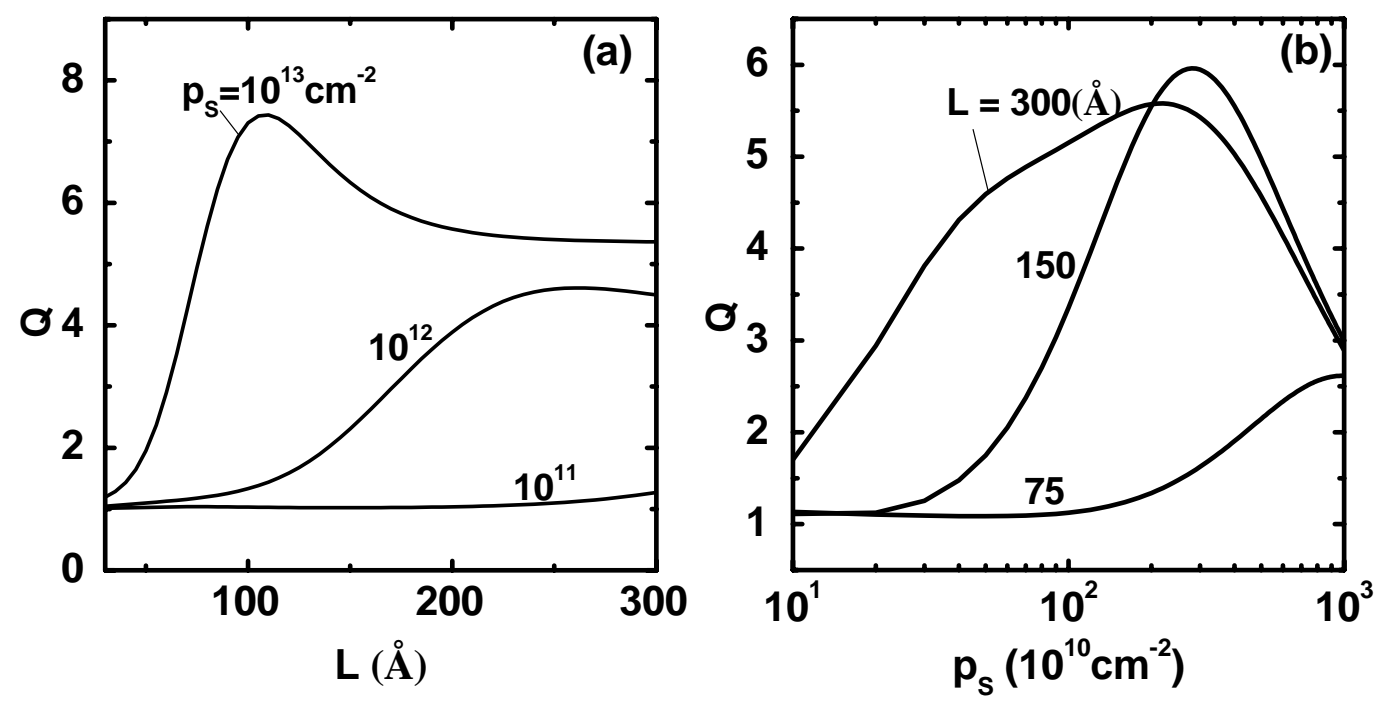

Fig. 1. Mobility enhancement factor $Q$ for the $p$-type square QW with a fixed correlation length $\Lambda=75 \AA$ vs (a) the well width $L$ for various sheet hole densities $p_{s}$ given on lines, and (b) the hole density $p_{s}$ for various well widths $L$ given on lines.

Next, we turn to the electron transport. It should be mentioned that the mobility of a two-dimensional electron gas (2DEG) in 2S doped square QWs was observed in Refs. [18] and [27] for the case where the well width and the sheet electron density were both varied. For 2DEG in the $\mathrm{Al}_{0.3} \mathrm{Ga}_{0.7} \mathrm{As} / \mathrm{GaAs} / \mathrm{Al}_{0.3} \mathrm{Ga}_{0.7} \mathrm{As} \mathrm{QW}$, the $4.2 \mathrm{~K}$ mobility was reported in Ref. [18] for the well widths (in units of $\AA$ ) and the electron densities (in units of $10^{11}$ $\left.\mathrm{cm}^{-2}\right)$ as follows: $\left(L, p_{s}\right)=(75,5.2),(85,6.7),(95,5.7),(110,6.9)$. As indicated from optical measurements, [18] in this relaxed QW with a spacer thickness $L_{s}=200 \AA$, SR scattering is the primary mechanism limiting the electron transport. The numerical result for 2DEG mobility is displayed in Fig. 2a for a roughness profile $\Delta=2.3 \AA, \Lambda=27 \AA$, where the data and the flat-band mobility are shown for comparison.

At last, for 2DEG in the GaSb/InAs/GaSb square QW, the $10 \mathrm{~K}$ mobility was reported in Ref. [27]. There, the used well widths (in units of $\AA$ ) and electron densities (in units of $\left.10^{12} \mathrm{~cm}^{-2}\right)$ were as follows: $\left(L, p_{s}\right)=(41.1,0.9),(53.6,1.2),(62.3,1.6),(72.7$, 1.5). For this QW of a small misfit $\epsilon_{\|}=0.006$, SR scattering was indicated to dominate the electron transport. [27,31] The numerical result for 2DEG mobility is displayed in Fig. $2 \mathrm{~b}$ for a roughness profile of $\Delta=3.2 \AA, \Lambda=15 \AA$, where the data and the flat-band mobility are also shown. 

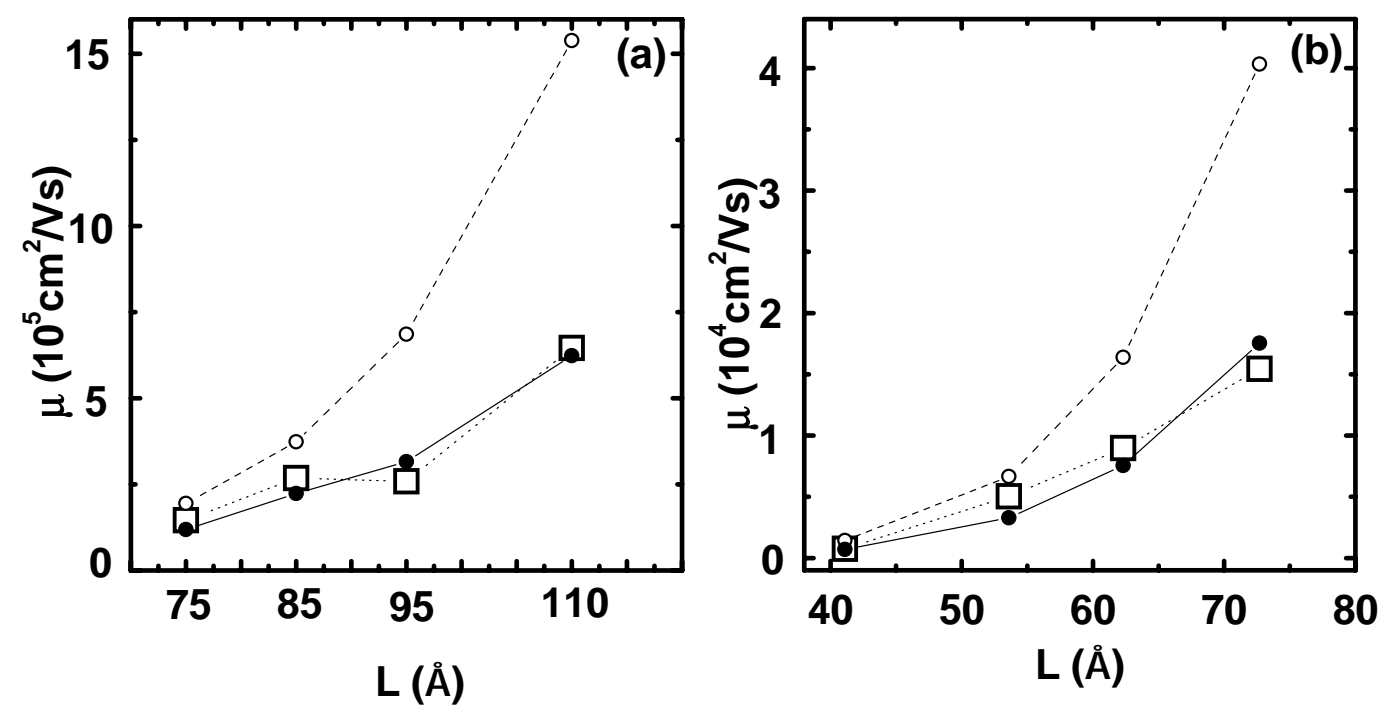

Fig. 2. 2DEG mobility $\mu$ in a symmetric $2 \mathrm{~S}$ doped square $\mathrm{QW}$ of $\mathrm{Al}_{0.3} \mathrm{Ga}_{0.7} \mathrm{As} / \mathrm{GaAs} / \mathrm{Al}_{0.3} \mathrm{Ga}_{0.7}$ As vs the well width $L$ with the electron densities $p_{s}$ varied as given in the text for an interface profile of $\Delta=2 \AA, \Lambda=15 \AA$. The solid and empty circles, and empty squares refer to the mobility in the bent-band and flat-band models, and the $4.2 \mathrm{~K}$ experimental data (Ref. [18]), respectively. The lines are a guide for the eyes (a). 2DEG mobility $\mu$ in a symmetric $2 \mathrm{~S}$ doped square QW of GaSb/InAs/GaSb vs the well width $L$ with the electron densities $p_{s}$ varied as given in the text for an interface profile of $\Delta=3.2 \AA, \Lambda=15 \AA$. The solid and empty circles, and empty squares refer to the mobility in the bent-band and flat-band models, and the $10 \mathrm{~K}$ experimental data (Ref. [27]), respectively. The lines are a guide for the eyes (b).

\section{REFERENCES}

[1] T. Ando, A. B. Fowler, and F. Stern, Rev. Mod. Phys. 54 (1982) 437.

[2] M. V. Fischetti and S. E. Laux, J. Appl. Phys. 80 (1996) 2234.

[3] T. Dziekan,P. Zahn, V. Meded, and S. Mirbt, Phys. Rev. B 75, 195213 (2007).

[4] M. V. Fischetti, F. Gámiz, and W. Hänsch, J. Appl. Phys. 92 (2002) 7320.

[5] J. E. Schirbir, I. J. Fritz, and L. R. Dawson, Appl. Phys. Lett. 46 (1985) 187.

[6] M. L. Lee, C. W. Leitz, Z. Cheng, A. J. Pitera, T. Langdo, M. T. Currie, G. Taraschi, E. A. Fitzgerald, and D. A. Antoniadis, Appl. Phys. Lett. 79 (2001) 3344.

[7] M. Myronov, T. Irisawa, S. Koh, O. A. Mironov, T. E. Whall, E. H. C. Parker, and Y. Shiraki, J. Appl. Phys. 97 (2005) 083701.

[8] R. E. Belford, B. P. Guo, Q. Xu, S. Sood, A. A. Thrift, A. Teren, A. Acosta, L. A. Bosworth, and J. S. Zell, J. Appl. Phys. 100 (2006) 064903.

[9] J. D. Sau and M. L. Cohen, Phys. Rev. B 75 (2007) 045208. 
[10] R. Dingle, H. L. Störmer, A. C. Gossard, and W. Wiegmann, Appl. Phys. Lett. 33 (1978) 665.

[11] T. Tsuchiya and T. Ando, Phys. Rev. B 48 (1993) 4599.

[12] G. Bastard, Wave Mechanics Applied to Semiconductor Heterostructures (Les Editions de Physique, Paris, 1988).

[13] R. M. Feenstra and M. A. Lutz, J. Appl. Phys. 78 (1995) 6091.

[14] D. N. Quang, V. N. Tuoc, N. H. Tung, and T. D. Huan, Phys. Rev. Lett. 89 (2002) 077601; Phys. Rev. B 68 (2003) 153306.

[15] D. N. Quang and N. H. Tung, Phys. Rev. B 77 (2008) 125335.

[16] D. N. Quang, N. H. Tung, D. T. Hien, and T. T. Hai, J. Appl. Phys. 104 (2008) 113711.

[17] Y. H. Xie, D. Monroe, E. A. Fitzgerald, P. J. Silverman, F. A. Thiel, and G. P. Watson, Appl. Phys. Lett. 63 (1993) 2263.

[18] K. L. Campman, H. Schmidt, A. Imamoglu, and A. C. Gossard, Appl. Phys. Lett. 69 (1996) 2554.

[19] H. Çelik, M. Cankurtaran, A. Bayrakli, E. Tiras, and N. Balkan, Semicon. Sci. Technol. 12 (1997) 389.

[20] N. Balkan, R. Gupta, M. Cankurtaran, H. Çelik, A. Bayrakli, E. Tiras, and M. Ç. Arikan, Superlattices Microstruct. 22 (1997) 263.

[21] M. Cankurtaran, H. Çelik, E. Tiras, A. Bayrakli, and N. Balkan, Phys. Status Solidi B 207 (1998) 139.

[22] R. J. H. Morris, T. J. Grasby, R. Hammond, M. Myronov, O. A. Mironov, D. R. Leadley, T. E. Whall, E. H. C. Parker, M. T. Currie, C. W. Leitz, and E. A. Fitzgerald, Semicond. Sci. Technol. 19 (2004) L106.

[23] C. Gerl, S. Schmult, H.-P. Tranitz, C. Mitzkus, and W. Wegscheider, Appl. Phys. Lett. 86 (2005) 252105.

[24] C. Gerl, S. Schmult, U. Wurstbauer, H.-P. Tranitz, C. Mizkus, and W. Wegscheider, Physica E 32 (2006) 258.

[25] B. Rössner, H. von Känel, D. Chrastina, G. Isella, and B. Batlogg, Thin Solid Films 508 (2006) 351.

[26] M. Myronov, K. Sawano, and Y. Shiraki, Appl. Phys. Lett. 88 (2006) 252115.

[27] F. Szmulowicz, S. Elhamri, H. J. Haugan, G. J. Brown, and W. C. Mitchel, J. Appl. Phys. 101 (2007) 04706.

[28] C. G. Van de Walle and R. M. Martin, Phys. Rev. B 34 (1986) 5621.

[29] A. Gold, Phys. Rev. B 35 (1987) 723; 38 (1988) 10798.

[30] A. Kahan, M. Chi, and L. Friedman, J. Appl. Phys. 75, 8012 (1994).

[31] A. Gold, J. Appl. Phys. 1033 (2008) 043718. 